\title{
A LONG-TERM ANALYSIS OF PASSENGER FLOWS ON A REGIONAL RAIL LINE
}

\author{
C. CAROPRESO, C. DI SALVO, M. BOTTE \& L. D'ACIERNO \\ Department of Civil, Architectural and Environmental Engineering, Federico II University of Naples, Italy.
}

\begin{abstract}
Promoting rail systems can represent a useful policy for rebalancing modal choices and reducing private car use, especially in high-density contexts. Obviously, an increase in passenger numbers is only possible if generalized costs (i.e. a weighted sum of times and monetary costs) associated to public transport are abated. According to the recent literature and current professional practice, most strategies for achieving this objective are based only on infrastructural interventions which may be unfeasible or inadequate in densely populated contexts. Likewise, the adoption of policies based on replacing existing fleets or reducing fare levels entails increases in national or regional subsidies, which would be difficult to achieve in the current economic climate.

Hence, our proposal is based on investigating effects on travel demand arising from the replacement or upgrading of existing signalling systems (both in terms of trackside and on-board equipment). Indeed, the recent European Union policy to create a single transnational interoperable rail network imposes the development of innovative signalling systems. In this context, since cost-benefit analysis has to be implemented to verify the economic and environmental feasibility of the proposed intervention strategy, an appropriate method should be developed to estimate passenger flows according to future configurations. In this article, we propose a method to determine travel demand in current and future contexts by appropriately processing data from Italy's national census on mobility, population growth forecasts and turnstile counts. The proposed approach is applied to the regional Naples-Sorrento rail line serving the metropolitan area of Naples in southern Italy in order to show its feasibility.

Keywords: environmental impacts, microscopic rail system simulation, public transport management, signalling system, travel demand estimation.
\end{abstract}

\section{INTRODUCTION}

Public transport, especially in the case of a rail-based system, may be considered a useful resource for managing travel demand and reducing the use of road (car and truck) systems. Obviously, since according to the assumptions of rational decision-maker (see, for instance, [1-5]) each user tends to choose the alternative of maximum utility (i.e. minimum disutility), road system costs need to be significantly increased and/or public transport costs considerably reduced.

Former strategies were based on the application of real pricing (such as road and parking pricing) or induced pricing (such as driving restrictions, restricted traffic zones and pedestrian areas). Details on the applications of such strategies and related implications can be found in Refs [6-11]. Subsequent policies were based on interventions to reduce travel times and ticket costs and improve service quality both in ordinary conditions and in disruption contexts (see Refs [12-23] for details). However, although the purpose of this article is to deal with the latter class of measures (i.e. improving public transport performance), it should

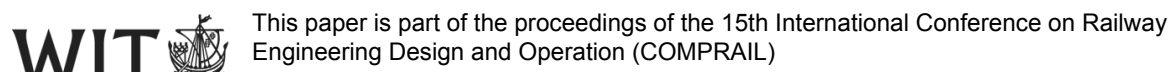

www.witconferences.com
} 
be pointed out that the literature also contains a third class of strategies based on reductions in social and environmental impacts of road systems, as reported by Refs [24-27].

The increase in public transport attractiveness can be obtained by reducing user generalized costs whose terms are the access and egress time (depending on station and stop location), the waiting time (depending on the headway between two successive rail convoys), the on-board travel time (depending on infrastructure features and rolling stock performance), the transfer time (depending on the layout of rolling stock, stations and platforms) and the ticket cost (depending on the adopted pricing scheme and subsidy policy). This means that improving interventions on a rail transport system are based on infrastructural measures (new lines or modification of existing lines), fleet improvement (partial or complete replacement of rolling stock) or signalling system modification (replacement or upgrade of trackside and on-board equipment). Although, especially in densely populated environments, the optimal intervention class consists in signalling system improvements, in some cases the infrastructural component may be essential in order to make any kind of further intervention effective. Obviously, in order to verify the benefits of each proposal it is necessary to simulate effects on travel demand explicitly (see, for instance, Refs [28-33]) for long-time horizons.

In this article we propose a methodology based on the use of different data sources (censuses, historical data, forecasts, counts, etc.) to estimate travel demand in a wide time period (several decades), taking into account variations due to infrastructure and signalling system interventions. The proposed approach was applied to a real regional line in southern Italy so as to show its feasibility. Hence, our proposal is organized as follows: in Section 2, we describe the general framework of the methodology for estimating travel demand; in Section 3, the application in the case of a regional rail line is proposed; finally, conclusions and research perspectives are summarized in Section 4.

\section{TRAVEL DEMAND DEFINITION}

Defining travel demand may be considered to be of prime importance in order to evaluate effects of any intervention on transportation systems. However, whatever the methodology adopted, the following requirements have to be met:

- accurate reproduction of the current situation;

- prediction of future conditions arising from demographic changes and/or different performance of transportation systems;

- travel demand must be considered a random variable and hence not only average values but also their distribution must be analysed.

This means that the model has to be elastic at least at the level of modal choice (in the case of transportation system variations) and trip generation (in the case of demographic changes).

Hence, in order to meet these needs, we propose the following methodology based on the use of different data sources. However, it should be pointed out that our proposal is based on Italian data sources even if generalization to a different context may easily be obtained.

The first phase consists in using data from the national census [34]. These data provide revealed information (i.e. related to behaviour actually occurring in the days prior to the survey) concerning mobility choices in terms of origin, destination, daily time period and transport mode. Census data concern systematic trips (i.e. for work or school purposes) during the average working day; origins and destinations are expressed in terms of municipalities; daily times are indicated as the morning peak hour (i.e. from 07:30 to 09:29) and the rest of the day. Obviously since trips are generally bidirectional (i.e. from home to the 
workplace and return), data provide only outward trips. In order to increase our dataset and satisfy the third requirement (i.e. a wide distribution of considered values), we propose to analyse data from at least two decades (i.e. data from the 2001 and 2011 Italian censuses). Finally, data related to our area of interest have to be selected by extracting from the whole national database only data related to internal trips (i.e. with origin and destination both in the study area) and exchange trips (i.e. with the origin or the destination in the study area).

The second phase consists in using data from mobility observatories (such as Ref. [35]) which provide further information such as total daily regional trips, rates of trips during morning peak hours, rates of trip chains (i.e. trips with intermediate destinations) and regional modal split. By combining these data it is possible to generate non-systematic trips during the average working day classified by origin and destination municipality, time period (i.e. peak hour or rest of the day), transport mode used and reference year (i.e. 2001 or 2011).

In the third phase, by using historical data from the resident population [36], data for systematic and non-systematic trips may be extended from the census period to a successive period by considering the trip generation model as elastic and adopting an increase or decrease rate equal to population variation (i.e. a variation in $\alpha \%$ of population in municipality $A$ provides a variation in $\alpha \%$ of all trips with origin in $A$ ).

The following phase consists in generating travel demand matrices related to all-day trips where the origin and destination are the stations of the rail line in question. This means that it is possible to identify two sub-phases: the first for obtaining round trips from outward trips in the case of all-day trips; the second for transforming trips expressed in terms of origin and destination municipalities into origin and destination stations.

The first sub-phase can be implemented by adopting the following formula:

$$
O D_{r t}^{i, m}=O D_{o t}^{i, m}+\left[O D_{o t}^{i, m}\right]^{\mathrm{T}}
$$

where $O D_{r t}^{i, m}$ is the origin-destination matrix related to round trips $(r t)$ throughout the day associated to purpose $i$ (i.e. systematic or non-systematic) and mode $m ; O D_{o t}^{i, m}$ is the origindestination matrix related to outward trips (ot) all day associated to purpose $i$ and mode $m$; $\left[O D_{o t}^{i, m}\right]^{\mathrm{T}}$ is the transposed matrix of $O D_{o t}^{i, m}$.

The second sub-phase consists in defining a regional network model in order to implement a minimum path approach for associating each municipality to each station. Obviously, if there are no stations in a municipality, we associate the nearest station; if there is only one station in a municipality, the problem is trivial (i.e. we associate the single station); finally, if there are two or more stations in a municipality, we hypothesize some distribution coefficients (for instance, related to turnstile counts).

The fifth phase consists in correcting origin-destination matrices associated to rail mode ( $r$ ) by using turnstile counts. Obviously since these counts are generally aggregated in a daily scale, we propose to correct the all-day matrices and modify initial matrices by adopting the same variation rates with the assumption that differences are related only to a different modal split (i.e. total travel demand is assumed as constant), that is,

$$
\begin{gathered}
O D_{r t}^{r}=\sum_{i} O D_{r t}^{i, r}, \\
\overline{O D_{r t}^{r}}=\underset{x \geq 0}{\arg \min } Z\left(d_{1}\left(x ; O D_{r t}^{r}\right) ; d_{2}\left(\Lambda(x) ; f^{r}\right)\right),
\end{gathered}
$$




$$
\begin{aligned}
& \delta_{j}=\overline{d_{j, r t}^{r}} / d_{j, r t}^{r} \text { with } \overline{d_{j, r t}^{r}} \in \overline{O D_{r t}^{r}} \text { and } d_{j, r t}^{r} \in O D_{r t}^{r} \text {, } \\
& \overline{O D_{h}^{i, r}}=\left\{\overline{d_{j, h}^{i, r}}: \overline{d_{j, h}^{i, r}}=d_{j, h}^{i, r} \cdot \delta_{j}\right\} \forall h \in[p h ; a d] \forall i \in[s ; n s], \\
& d_{j, h}^{i}=\sum_{m} d_{j, h}^{i, m} \\
& \overline{O D_{h}^{i, m}}=\left\{\overline{d_{j, h}^{i, m}}: \overline{d_{j, h}^{i, m}}=d_{j, h}^{i, m} \cdot\left(d_{j, h}^{i}-\overline{d_{j, h}^{i, r}}\right) / \sum_{m \neq r} d_{j, h}^{i, m}\right\} \\
& \forall m \neq r \quad \forall h \in[p h ; a d] \quad \forall i \in[s ; n s],
\end{aligned}
$$

where $O D_{r t}^{r}$ is the origin-destination matrix related to all-day round trips ( $r t$ ) associated to mode $r$ (i.e. rail mode), $\overline{O D_{r t}^{r}}$ is the correction of matrix $O D_{r t}^{r} ; x$ is the variable expressing in the optimization problem (3) the generic value of matrix $O D_{r t}^{r} ; Z(\cdot)$ is the objective function to be minimized; $d_{1}$ is a function which expresses the distance between matrix $x$ and the a-priori estimation of matrix $O D_{r t}^{r} ; \Lambda(\cdot)$ is the assignment function which provides passenger flows associated to origin-destination matrix $x ; f^{r}$ is the vector of turnstile counts; $d_{1}$ is a function which expresses the distance between flows obtained by assigning matrix $x$ and flows provided by turnstile counts (i.e. $f^{r}$ ); $\delta_{j}$ is the variation rate of travel demand associated to origindestination $j ; \overline{d_{j, r t}^{r}}$ is the generic element of matrix $\overline{O D_{r t}^{r}} ; d_{j, r t}^{r}$ is the generic element of matrix $O D_{r t}^{r} ; \overline{O D_{h}^{i, m}}$ is the corrected origin-destination matrix in the time period $h$, for purpose $i$ by using mode $m ; \overline{d_{j, h}^{i, m}}$ is the generic element of matrix $\overline{O D_{h}^{i, m}}$ associated to origin-destination $j ; d_{j, h}^{i, m}$ is the a-priori estimation of trips in the case of origin-destination $j$, in the time period $h$, for purpose $i$ by using mode $m ; d_{j, h}^{i}$ is the a-priori estimation of trips in the case of origindestination $j$, in the time period $h$, for purpose $i$ by using all transportation mode.

It is worth noting that since variable $i$, expressing the purpose of the trip, may assume $s$ for systematic and $n s$ for non-systematic and variable $h$, expressing time period, may assume $p h$ for the morning peak hour and $a d$ for all day, we obtain that, in the case of corrected matrices, eqn (1) can be expressed as follows:

$$
\overline{O D_{r t}^{i, m}}=\overline{O D_{a d}^{i, m}}+\left[\overline{O D_{a d}^{i, m}}\right]^{\mathrm{T}},
$$

where $\overline{O D_{r t}^{i, m}}$ is the corrected origin-destination matrix related to all-day round trips $(r t)$ associated to purpose $i$ and mode $m$.

The sixth phase consists in the temporal extension to one or more analysis periods of corrected matrices. In particular, the new matrices may be obtained by considering (real or estimated) demographic variations as in the case of the third phase.

In order to make demand elastic at least at modal choice level, it is possible to specify, calibrate and validate a suitable choice model by adopting traditional methodology proposed in the literature (see, for instance, Ref. [4]). In particular, it is necessary to:

- specify a utility formulation and a probability choice model such as: 


$$
\begin{gathered}
V_{j, h}^{i, m}=V_{j, h}^{i, m}\left(\beta_{k}^{m}\right)=\sum_{k} \beta_{k}^{m} \cdot X_{k, j, h}^{i, m}, \\
p_{j, h}^{i}[m]=p_{j, h}^{i}[m]\left(V_{j, h}^{i, m}\left(\beta_{k}^{m}\right)\right)=p_{j, h}^{i}[m]\left(\beta_{k}^{m}\right),
\end{gathered}
$$

where $V_{j, h}^{i, m}$ is the utility associated to mode $m$ in the case of purpose $i$ during the time period $h$ for travelling between the origin-destination $j ; \beta_{k}^{m}$ is the parameter associated to $k$ th attribute of the mode $m ; X_{j, h}^{i, m}$ is the $k$ th attribute associated to mode $m$ in the case of purpose $i$ during the time period $h$ for travelling between the origin-destination $j ; p_{j, h}^{i}[m]$ is the probability of choosing mode $m$ for travelling between the origin-destination $j$ in the case of purpose $i$ during the time period $h$;

- calibrate the values of parameters $\beta_{k}^{m}$ by solving the following optimization problem:

$$
\hat{\beta}_{k,}^{m}=\underset{\beta_{k}^{m}}{\arg \max }\left(\ln L\left(\beta_{k}^{m}\right)\right)=\sum \ln \left(p_{j, h}^{i}[m]\left(\beta_{k}^{m}\right)\right),
$$

where $\hat{\beta}_{k}^{m}$ is a calibrated value of parameter $\beta_{k}^{m} ; L(\cdot)$ is a likelihood function to be maximized;

- validate the results by means of suitable statistical tests.

The following phase consists in determining hourly matrices being consistent with the corrected matrices, that is:

- in the time period 07:30-09:29, the hourly travel demand can be obtained by dividing by 2 the peak hour origin-destination matrix $\overline{O D_{p h}^{i, r}}$;

- in the morning period (for instance, until 13:30), except in the peak hour period already analysed, the hourly travel demand can be obtained by dividing the outward matrix minus the peak hour matrix (i.e. $\overline{O D_{a d}^{i, r}}-\overline{O D_{p h}^{i, r}}$ ) by adopting suitable coefficients (for instance, derived from previous flow studies);

- in the afternoon and evening period (for instance, from 13:30 onwards), hourly demand may be obtained by dividing the transposed of the outward matrix $\overline{O D_{a d}^{i, r}}$ by adopting suitable coefficients.

In order to consider interaction between travel demand and the rail service, we suggest adopting the approach proposed by D'Acierno et al. [17, 29] which consists in considering explicitly the behaviour of passengers waiting on a platform when a train arrives. The methodology allows boarding, alighting and on-board passenger flows to be determined for each train, average passenger travel and waiting times for each time period and hence the total generalized cost for passengers on the rail system.

The application of the interaction model for each analysed scenario allows all terms of the objective function to be calculated, that is,

$$
\Psi=F C+U C+E C,
$$

with

$$
F C=O C-T R
$$




$$
\begin{aligned}
& U C=R P C+M T P C+R C, \\
& R P C=T T C+W T C+T C,
\end{aligned}
$$

where $\Psi$ is the objective function to minimize; $F C$ are the total costs of the firm operating the rail system; $U C$ are the user generalized costs; $E C$ are the external costs; $O C$ are the operative costs of the analysed rail system; $T R$ are the traffic revenues due to the sale of rail tickets; $R P C$ are the passenger costs on the analysed rail system; $M T P C$ are the passenger costs on mass-transit systems except the analysed rail system; $R C$ are the user costs on the road system; TTC are the passenger costs associated to travel times; WTC are the passenger costs associated to waiting times; $T C$ are passenger monetary costs. Details on the calculation of these terms can be found in Refs [4, 17, 19, 37].

Obviously, in order to calculate cost terms in the case of other mass-transit and road systems it is necessary to adopt a multimodal approach which consists in generating a complete public transport and private car network in order to calculate flows and related time and monetary costs (see, for instance, Refs [4, 7, 19, 37-39]).

\section{REGIONAL LINE APPLICATION}

In order to show the feasibility of the proposed methodology, we applied it in the case of a real regional rail line, namely the Naples-Sorrento line which connects the regional capital (i.e. Naples) with the Sorrento peninsula (the city of Sorrento represents the line terminus) in the south of Italy.

As shown in Fig.1, the line, which is $41.5 \mathrm{~km}$ long, can be decomposed into four sections: the Naples-Barra double-track section, $4.5 \mathrm{~km}$ long, at the end of which there is a branch to Sarno; the Barra-Torre Annunziata double-track section, $15.6 \mathrm{~km}$ long, at the end of which there is a branch to Poggiomarino; the Torre Annunziata-Moregine double-track section, $4.4 \mathrm{~km}$ long, after which there is a single-track section; and the Moregine-Sorrento single-track section, $17.0 \mathrm{~km}$ long, which finishes with the line terminus. Therefore, in the first section there is the overlap of three lines (Naples-Sorrento, Naples-Poggiomarino and Naples-Sarno), in the second section the interference is limited to only two lines and in the third section there are bottlenecks related to the Moregine-Sorrento single-track section.

The application consists in verifying the effects of a different signalling system in terms of variation in the objective function. In the case of a cost-benefit analysis, intervention costs have to be compared with global benefits. Hence, since we want to identify the improving signalling system in terms of performance and not in terms of existing commercial products, it is necessary to calculate long-term benefits so as to define cost thresholds for implementing new technologies.

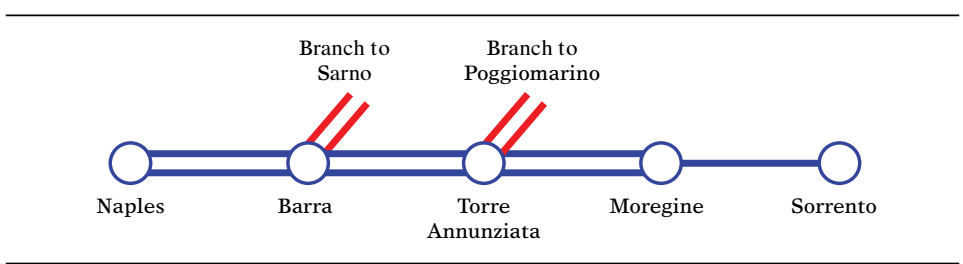

Figure 1: Functional scheme of the Naples-Sorrento line. 
However, since the last line section is single track, in order to improve line performance and maximize the effects of a different signalling system, we also explored the possibility of an infrastructural intervention consisting in doubling the line (i.e. construct another track in order to make the line double track). Therefore, in the application, we considered the following seven scenarios:

- Scenario 1 consists in considering the current framework of the line in terms of infrastructure, signalling system and timetable;

- Scenario 2 consists in considering the current framework of the line in terms of infrastructure and signalling system while, in terms of timetable, we maximize the number of runs on the Naples-Sorrento line by considering the timetable of other overlapping lines as invariant;

- Scenario 3 consists in considering the same assumption as Scenario 2 but considering the Naples-Sorrento line as having priority over the other overlapping lines (i.e. a run of the other lines can be done only if a suitable time-window is available);

- Scenario 4 consists in doubling the 'Moregine-Sorrento' section so as to make the whole Naples-Sorrento line double track. In this case, we consider the existing signalling system as invariant and the timetable optimized by maximising the number of runs and considering the timetable of other overlapping lines as invariant;

Table 1: Objective function values.

\begin{tabular}{lccccc}
\hline \multirow{2}{*}{ Scenario } & \multicolumn{5}{c}{ Analysed year } \\
& 2016 & 2026 & 2036 & 2046 & 2056 \\
\hline 1 & $26,001,375$ & $25,470,409$ & $24,640,361$ & $23,484,308$ & $21,984,231$ \\
2 & $25,966,660$ & $25,438,469$ & $24,612,758$ & $23,462,747$ & $21,970,508$ \\
3 & $25,962,951$ & $25,434,976$ & $24,609,605$ & $23,460,065$ & $21,968,439$ \\
4 & $25,822,162$ & $25,301,345$ & $24,487,163$ & $23,353,207$ & $21,881,802$ \\
5 & $25,694,677$ & $25,178,209$ & $24,370,825$ & $23,246,337$ & $21,787,218$ \\
6 & $25,030,696$ & $24,533,087$ & $23,755,185$ & $22,671,758$ & $21,265,919$ \\
7 & $22,935,558$ & $22,488,373$ & $21,789,297$ & $20,815,657$ & $19,552,276$ \\
\hline
\end{tabular}

Table 2: Variations in the objective function.

\begin{tabular}{lccccc}
\hline \multirow{2}{*}{ Scenario } & \multicolumn{5}{c}{ Analysed year } \\
& 2016 & 2026 & 2036 & 2046 & 2056 \\
\hline 1 & - & - & - & - & - \\
2 & $-0.14 \%$ & $-0.13 \%$ & $-0.12 \%$ & $-0.10 \%$ & $-0.06 \%$ \\
3 & $-0.16 \%$ & $-0.15 \%$ & $-0.13 \%$ & $-0.11 \%$ & $-0.07 \%$ \\
4 & $-0.74 \%$ & $-0.71 \%$ & $-0.67 \%$ & $-0.60 \%$ & $-0.49 \%$ \\
5 & $-1.26 \%$ & $-1.22 \%$ & $-1.16 \%$ & $-1.08 \%$ & $-0.95 \%$ \\
6 & $-3.93 \%$ & $-3.87 \%$ & $-3.78 \%$ & $-3.63 \%$ & $-3.42 \%$ \\
7 & $-12.20 \%$ & $-12.11 \%$ & $-11.96 \%$ & $-11.73 \%$ & $-11.40 \%$ \\
\hline
\end{tabular}


- Scenario 5 consists in considering the same assumption as Scenario 4 but considering the Naples-Sorrento line as a priority over the other overlapping lines (i.e. a run on other lines can be undertaken only if a suitable time-window is available);

- Scenario 6 is similar to Scenario 5 but considers a new signalling system which allows a headway of 3 minutes between two successive rail convoys;

- Scenario 7 is similar to Scenario 6 but considers a headway of 2 minutes between two successive rail convoys.

The application of the proposed methodology in the case of the above seven scenarios is summarized in Tables 1 and 2.

Numerical results show that only the timetable optimization (i.e. Scenarios 2 and 3) provides limited improvements, which are lower than $0.2 \%$. In this context, complete replacement of the signalling system is not sufficient to improve line performance since the real issue is the single-track section. For these reasons, although simple doubling of the line (Scenarios 4 and 5) provides even limited improvements $(<1.5 \%)$, it allows us to benefit fully from creating a different signalling system which allows the current minimum headway of 4.62 minutes to be modified.

\section{CONCLUSIONS AND RESEARCH PROSPECTS}

The analysis of any transportation system requires implementation of a cost-benefit approach where long-term estimation of travel demand is a major requirement. In this context we proposed a method based on the use of different source data and applied it in the case of a regional rail line.

Analyses showed that, in the context considered, the existence of a single-track section represents the main limitation in line improvements. However, the only infrastructural intervention, whose costs are about $€ 300-800$ million, does not provide significant improvements unless coupled with the replacement of the signalling system.

In terms of future research, we suggest applying the proposed methodology, based on data from Italian sources, in other contexts both vis-à-vis other Italian railways (in order to verify the reliability of the adopted datasets) and other non-Italian railways (in order to test the methodology in the case of different source data).

\section{ACKNOWLEDGEMENTS}

This article is partially supported under research project FERSAT grant no. PON03PE_00159_4.

\section{REFERENCES}

[1] Domencich, T.A. \& McFadden, D., Urban Travel Demand: A Behavioural Analysis, American Elsevier: New York, 1975.

[2] Ben-Akiva, M. \& Lerman, S.R., Discrete Choice Analysis: Theory and Application to Travel Demand. The MIT Press: Cambridge, MA, 1985.

[3] Cascetta, E., Pagliara, F. \& Papola, A., Alternative approaches to trip distribution modelling: A retrospective review and suggestions for combining different approaches. $\mathrm{Pa}$ pers in Regional Science, 86(4), pp. 597-620, 2007.

[4] Cascetta, E., Transportation Systems Analysis: Models and Applications. Springer: New York, 2009.

[5] Pagliara, F. \& Timmermans H.J.P., Choice set generation in spatial contexts: A review, Transportation Letters: The International Journal of Transportation Research, 1(3), pp. 181-196, 2009. 
[6] Ho, H.W., Wong, S.C., Yang, H. \& Loo, B.P.Y., Cordon-based congestion pricing in a continuum traffic equilibrium system. Transportation Research Part A, 39(7-9), pp. 813-834, 2005.

[7] D’Acierno, L., Ciccarelli, R., Montella, B. \& Gallo, M., A multimodal multiuser approach for analysing pricing policies in urban contexts. Journal of Applied Sciences, 11(4), pp. 599-609, 2011.

[8] Ibeas, A., Cordera, R., dell'Olio, L. \& Moura, J.L., Modelling demand in restricted parking zones. Transportation Research Part A, 45(6), pp. 485-498, 2011.

[9] Biggiero, L., The impact of transport management on the local activities system: The role of limited traffic zones. WIT Transactions on the Built Environment, 138, pp. 669-678, 2014.

[10] Noordegraaf, D.V., Annema, J.A. \& van Wee, B., Policy implementation lessons from six road pricing cases. Transportation Research Part A, 59, pp. 172-191, 2014.

[11] Wang, L., Xu, J. \& Qin, P., Will a driving restriction policy reduce car trips? The case study of Beijing, China. Transportation Research Part A, 67, pp. 279-290, 2014.

[12] Brännlund, U., Lindberg, P.O., Nõu, A. \& Nilsson, J.E., Railway timetabling using Lagrangian relaxation. Transportation Science, 32(4), pp. 358-369, 1998.

[13] Goverde, R.M.P., Punctuality of railway operations and timetable stability analysis. PhD Thesis, TU Delft, The Netherlands, 2005.

[14] D’Ariano, A., Corman, F., Pacciarelli, D. \& Pranzo, M., Reordering and local rerouting strategies to manage train traffic in real time. Transportation Science, 42(4), pp. 405-419, 2008.

[15] D’Ariano, A., Pacciarelli, D. \& Pranzo, M., Assessment of flexible timetables in real-time traffic management of a railway bottleneck. Transportation Research Part $C$, 16(2), pp. 232-245, 2008.

[16] Canca, D., Zarzo, A., Algaba, E. \& Barrena, E., Confrontation of different objectives in the determination of train scheduling. Procedia: Social and Behavioral Sciences, 20, pp. 302-312, 2011.

[17] D'Acierno, L., Gallo, M., Montella, B. \& Placido, A., The definition of a model framework for managing rail systems in the case of breakdowns. Proceedings of the 16th IEEE Conference on Intelligent Transportation Systems (ITSC), The Hague, The Netherlands, pp. 1059-1064, 2013. DOI: 10.1109/ITSC.2013.6728372.

[18] Quaglietta, E. Corman, F. \& Goverde, R.M.P., Impact of a stochastic and dynamic setting on the stability of railway dispatching solutions. Proceedings of the 16th IEEE Conference on Intelligent Transportation Systems (ITSC), The Hague, The Netherlands, pp. 1035-1040, 2013. DOI: 10.1109/ITSC.2013.6728368.

[19] Montella, B., D'Acierno, L. \& Gallo, M., A multimodal approach for determining optimal public transport fares. Journal of Applied Sciences, 14(21), pp. 2767-2781, 2014.

[20] Gallo, M., Simonelli, F., De Luca, G. \& De Martinis, V., Estimating the effects of energy-efficient driving profiles on railway consumption. Proceedings of the 15th IEEE International Conference on Environment and Electrical Engineering (EEEIC), Rome, Italy, pp. 1059-1064, 2015. DOI: 10.1109/EEEIC.2015.7165269.

[21] Biggiero, L., Pagliara, F., Patrone, A. \& Peruggini, F., Spatial equity and high-speed rail systems. WIT Transactions on the Built Environment, 164, 2016.

[22] Botte, M., Di Salvo, C., Placido, A., Montella, B. \& D’Acierno, L., A Neighbourhood Search Algorithm (NSA) for determining optimal intervention strategies in the case of metro system failures. International Journal of Transport Development and Integration, 1 (1), pp. 63-73, 2017. 
[23] D’Acierno, L., Placido, A., Botte, M. \& Montella, B., A methodological approach for managing rail disruptions with different perspectives. International Journal of Mathematical Models and Methods in Applied Sciences, 10, pp. 80-86, 2016.

[24] Dell'Acqua, G., Russo, F. \& Biancardo S.A., Risk-type density diagrams by crash type on two-lane rural roads. Journal of Risk Research, 16(10), pp. 1297-1314, 2013.

[25] Dell'Acqua, G., Modeling driver behavior by using the speed environment for two-lane rural roads. Transportation Research Record, 2472, pp. 83-90, 2015.

[26] Bifulco, G.N., Cantarella, G.E., Simonelli, F. \& Velonà, P., Advanced traveller information systems under recurrent traffic conditions: Network equilibrium and stability. Transportation Research Part B, 2016. DOI: 10.1016/j.trb.2015.12.008.

[27] Pariota, L., Bifulco, G.N. \& Brackstone, M., A linear dynamic model for driving behavior in car following. Transportation Science, 2016. DOI: 10.1287/trsc.2015.0622.

[28] Lo, H.-P. \& Chan, C.-P., Simultaneous estimation of an origin-destination matrix and link choice proportions using traffic counts. Transportation Research Part A, 37(9), pp. 771-788, 2003.

[29] D’Acierno, L., Gallo, M., Montella, B. \& Placido, A., Analysis of the interaction between travel demand and rail capacity constraints. WIT Transactions on the Built Environment, 128, pp. 197-207, 2012.

[30] Cascetta, E., Papola, A., Marzano, V., Simonelli, F. \& Vitiello, I., Quasi-dynamic estimation of o-d flows from traffic counts: Formulation, statistical validation and performance analysis on real data. Transportation Research Part B, 55, pp. 171-187, 2013.

[31] Ercolani, M., Placido, A., D'Acierno, L. \& Montella, B., The use of microsimulation models for the planning and management of metro systems. WIT Transactions on the Built Environment, 135, pp. 509-521, 2014.

[32] Cantarella, G.E., de Luca, S. \& Cartenì, A., Stochastic equilibrium assignment with variable demand: theoretical and implementation issues. European Journal of Operational Research, 241(2), pp. 330-347, 2015.

[33] Di Mauro, R., Botte, M. \& D’Acierno, L., An analytical methodology for extending passenger counts in a metro system. International Journal of Transport Development and Integration, 1, 2016.

[34] Istituto Nazionale di Statistica - ISTAT (Italian National Institute of Statistics). Population and Housing Census, http://www.istat.it/it/censimento-popolazione (last accessed: March 2016).

[35] Osservatorio sui comportamenti di mobilità degli italiani - AudiMob (Observatory on the Italian mobility behaviour). Regional Mobility Statistics, http://www.isfort.it/sito/ statistiche/Audimob.htm (last accessed: March 2016).

[36] Istituto Nazionale di Statistica - ISTAT (Italian National Institute of Statistics). Resident Population, http://www.istat.it/it/popolazione (last accessed: March 2016).

[37] Gallo, M., Montella, B. \& D'Acierno, L., The transit network design problem with elastic demand and internalisation of external costs: An application to rail frequency optimisation. Transportation Research Part C, 19(6), pp. 1276-1305, 2011.

[38] D’Acierno, L., Montella, B. \& Gallo, M. A fixed-point model and solution algorithms for simulating urban freight distribution in a multimodal context. Journal of Applied Sciences, 11(4), pp. 647-654, 2011.

[39] D'Acierno, L., Gallo, M. \& Montella, B., A theoretical multimodal assignment model for simulating intermodal trips. WIT Transactions on the Built Environment, 116, pp. 17-29, 2011. 